\title{
On certain new connections between Legendre and Bessel Functions
}

By S. C. Mitra, Dacca, India.

(Received 14th November, 1934. Read 7th December, 1934.)

Let $n$ be a positive integer. Then we know that ${ }^{1}$, if $m>-1$,

$$
\int_{0}^{1} P_{n}\left(1-2 y^{2}\right) y^{2 m+1} d y=\frac{1}{2}(-1)^{n} \frac{\{\Gamma(m+1)\}^{2}}{\Gamma(m-n+1) \Gamma(m+n+2)} \text {. }
$$

Consider the integral

which is equal to ${ }^{2}$

$$
I=\int_{0}^{1} P_{n}\left(1-2 y^{2}\right) J_{0}(2 y z) y d y,
$$

$$
\sum_{m=0}^{\infty} \frac{(-1)^{m} z^{2 m}}{\{\Gamma(m+1)\}^{2}} \int_{0}^{1} P_{n}\left(1-2 y^{2}\right) y^{2 m+1} d y .
$$

On integrating term by term, we get

$$
\begin{aligned}
I & =\frac{1}{2}(-1)^{n} \sum_{m=0}^{\infty} \frac{(-1)^{m} z^{2 m}}{\Gamma(m-n+1) \Gamma(m+n+2)} \\
& =\frac{1}{2} \sum_{m=0}^{\infty} \frac{(-1)^{m} z^{2 m+2 n}}{\Gamma(m+1) \Gamma(m+2 n+2)} \\
& =(2 z)^{-1} J_{2 n+1}(2 z) .
\end{aligned}
$$

In a similar manner, we can prove the following results:

$$
\begin{aligned}
& \int_{0}^{1} P_{n}\left(1-2 y^{4}\right) J_{0}(2 y z) I_{0}(2 y z) y^{3} d y \\
& =(8 z)^{-1} \frac{d}{d z}\left\{J_{2 n+1}(2 z) I_{2 n+1}(2 z)\right\} \\
& \int_{0}^{1} P_{n}\left(1-2 y^{4}\right) \frac{d}{d y}\left[y^{2}\left\{\operatorname{ber}_{1}^{2}(2 y z)+\operatorname{bei}_{1}^{2}(2 y z)\right\}\right] d y \\
& =(-1)^{n}\left\{\operatorname{ber}_{2 n+1}^{2}(2 z)+\operatorname{bei}_{2 n+1}^{2}(2 z)\right\}
\end{aligned}
$$

and

$$
\begin{gathered}
\int_{0}^{1} P_{n}\left(1-2 y^{4}\right) \frac{d}{d y}\left\{y^{2} J_{1}(2 y z) I_{1}(2 y z)\right\} d y \\
=J_{2 n+1}(2 z) I_{2 n+1}(2 z)
\end{gathered}
$$

1 Equation (1) follows at once by putting $x=1-2 y^{2}$, using Rodrigues' formula for $P_{n}(x)$, and integrating $n$ times by parts. Cf. Cooke, Proc. London Math. Soc., 23 (1924), xix, equ. (3).

2 The process of arrangement and term by term integration can be easily justified. 